\section{Stellar evolution}

\section{R. J. Tayler}

Stellar Evolution. Second edition. By A. J. Meadows. Pp. 171. (Pergamon: Oxford, 1978.) Hardback £7.50; paperback $£ 2.50$.

THE subject of stellar structure and evolution is believed to be the branch of astrophysics which is best understood. Because most parts of physicssuch as nuclear physics, atomic physics, thermodynamics and statistical physics, electromagnetism and gravitation-are involved in determining the structure of a star, it is a very suitable topic in applied physics to introduce to undergraduates. It is also possible to present the subject to an audience with a slighter knowledge of physics and this is the concern of Professor Meadows.

When the first edition of this book was published in 1967 , it received a warm welcome because of the manner in which it bridged the gap between very superficial discussions of stellar structure and evolution in books for the layman and complex mathematical texts for the professional astronomer. Twenty years earlier George Gamow's book The Birth and Death of the Sun had served a similar purpose, but by 1967 this was seriously out of date in detail. The first edition of Meadows' book gave a clear account of the observed properties of stars and the manner in which they are obtained. It then discussed the principles underlying the structure of stars, the source of the energy radiated from stellar surfaces, the manner in which this energy travels from the centre to the surface of a star, and the physical state of the matter inside stars. The remainder of the book was concerned with the evolution of stars from their birth as dense condensations in interstellar gas clouds, through the major part of their life as main sequence stars (the present state of the Sun) and subsequently as red giants, to their death as white dwarfs, possibly after a supernova explosion. It was then difficult to see how our knowledge of stellar evolution could be treated in a significantly clearer manner without the introduction of mathematical detail, which was completely absent from Meadows' book.

The first edition has now been revised to take account of developments in the subject. Most of the book has been photographically reproduced from the first edition and in most places it is possible to sec where changes have been made because of a slight variation in contrast of the type. In the first half of the book there are only a small number of detailed alterations but later there are much more substantial changes, particularly in chapters 6,7 and 8 . The book is essentially unchanged in length, the addition of new material being balanced by the removal of speculative or unimportant material.

Substantial changes in the new edition include the following. A section has been added on the solar neutrino experiment, the results of which are at present the major question mark against the standard view of stellar structure. There is a completely revised discussion of the evolution of low mass stars following the helium flash, the explosive onset of the conversion of helium into carbon. The major change concerns stellar old age and death. Shortly after the first edition appeared, pulsars were discovered and it was

\section{Microcosm of astrophysics}

\section{F. Graham Smith}

The Crab Nebula. By Simon Mitton. Pp. 194. (Faber and Faber: London; Scribners: Totowa, New Jersey, 1979.) f6.50; $\$ 14.95$.

THE 900th birthday of the Crab Nebula was celebrated by radio astronmers in July 1954. At that time it was almost the only celestial object, apart from the Sun, where a close link between optical and radio astronomy had emerged. In these days, when such links have appeared throughout astronomy, the Crab still has a unique importance, and annual celebrations of the supernova explosion would still be appropriate. Geoffrey Burbridge once said that astronomy could be divided into two parts: the astronomy of the Crab Nebula and the astronomy of everything else. There are indeed links between the Crab and almost everything eise in astronomy, so that a discourse on the Crab can be used as an introduction, for example, to astronomy, stellar evolution, cosmology and interstellar matter; including the Crab Pulsar extends the list to the physics of condensed matter, high energy electrodynamics, superconductivity, and many other aspects of quantum physics.

This microcosm of astrophysics can thereforc be uscd as a general introduction to astronomy, which should appeal to any scientist and which may persuade some that astronomy is a very useful means of education in basic physics. Popular books on astronomy soon realised that they are neutron stars. It now seems certain that neutron stars rather than white dwarfs are a normal end-product of a supernova explosion. In addition it has been realised that sufficiently massive stellar remnants will be neither white dwarfs nor neutron stars but will be black holes. Rather surprisingly in view of the great current interest in close binary stars and mass exchange between their components, the section on this topic has been slightly reduced in length, although there is a brief mention of the possible detection of a black hole in Cygnus X-1.

The revised edition of this book remains a very good non-mathematical introduction to stellar evolution and the paperback edition is very reasonably priced.

R. J. Tayler is Professor of Astronomy at the University of Sussex, Brighton, UK.

often do not succeed in presenting the more serious aspects of the subject, and their readers might not realise the important part which is played by astronomy in postgraduate physics education. Mitton is a physicist, and emphasises the basic physics throughout, but his book is nevertheless a popular book. $\mathrm{He}$ eschews mathematics, and uses not a single equation. The reader need only be moderately numerate, so that he can appreciate the large powers of ten involved in any branch of astrophysics.

The decision to exclude mathematics reduces the astronomy of the Crab to less than half of the total of astronomy; and further choices have been made. Mitton has chosen to make the book readable, straight through, for anyone with an undergraduate level of physics or astronomy. Others, with less expert knowledge, will enjoy at least the historical accounts of the supernova explosion, gleaned from Chinese and Japanese records of the "guest star", and the more recent history of discoveries-notably of the pulsar which remains the centre of the nebula, providing the powerhouse for the energy to keep the nebular shining. For the physicist, the exposition of the basic concepts of degeneracy in white dwarfs and neutron stars, and of superconductivity, will have a strong appeal, as they show most clearly the connection between terrestrial and celestial solidstate physics.

Mitton is an enthusiast. In conveying his enthusiasm he does sometimes overstep the mark, in such purple passages as his description of synchrotron radiation: "photon screeches from very energetic electrons as they wrap themselves round the ambient magnetic field". He refers to the giant pulses from the pulsar as "jumbn", he tells us 